\title{
(ப)
}

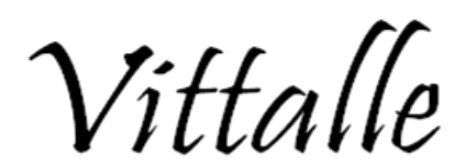

FURG

ISSN $2177-7853$

\section{A eficácia do tratamento da obesidade através do transplante da microbiota fecal de indivíduos magros}

\author{
Júlia de Oliveira Penteado ${ }^{\mathrm{a}, \mathrm{b}^{*}}$, Rúbia Gabriela Fernandes Salgado ${ }^{\mathrm{b}}$, Edson Barlem ${ }^{\mathrm{b}, \mathrm{c}}$ \\ aLaboratório de Ensaios Farmacológicos e Toxicológicos -Instituto de Ciências Biológicas. \\ Universidade Federal de Rio Grande - FURG \\ bPrograma de Pós-Graduação em Ciências da Saúde -Faculdade de Medicina - \\ Universidade Federal do Rio Grande - FURG, Rio Grande, RS, Brasil \\ ${ }^{\mathrm{c}}$ Escola de Enfermagem - Universidade Federal de Rio Grande - FURG
}

\section{Histórico do Artigo}

Recebido em: 30/09/2016

Aceito em: 04/12/2016

Palavras-chave

Doenças gastrintestinais

Firmicutes

Bacterioides

Keywords

Gastrientinal diseases

Firmicutes

Bacterioides

*Autor correspondente: julia-penteado@hotmail.com (J. O. Penteado)

\section{RESUMO}

Há um crescente interesse na compreensão do papel da microbiota intestinal para elucidar o seu potencial terapêutico. Uma das possíveis aplicações do transplante da microbiota fecal é no tratamento da obesidade, também denominado bacterioterapia fecal. Dessa forma, o objetivo do trabalho foi compreender o efeito terapêutico da microbiota intestinal na saúde do indivíduo. Assim como, conhecer a aplicação do transplante da microbiota fecal de indivíduos magros em indivíduos obesos. Tratou-se de uma revisão integrativa da literatura. A busca dos artigos foi realizada em abril de 2016, no Portal Regional da Biblioteca Virtual da Saúde, no período 2006-2016. Foram utilizados como critérios de inclusão: artigos disponíveis na íntegra de acesso livre; que abordaram a temática do estudo; apresentação de resumo; em língua portuguesa, inglesa ou espanhola. Dentre os descritores de ciências da saúde, utilizou-se "gastrointestinal microbiome", "fecal microbiota transplantation" e "obesity". Os artigos apresentaram como principal resultado mais encontrado que o transplante da microbiota fecal do indivíduo magro para o indivíduo obeso pode ser usado no tratamento de várias doenças tais como infecções intestinais ou em Diabetes Mellitus. Portanto, faz-se necessário a divulgação do tema em um âmbito multiprofissional facilitando o aumento de pesquisas em todas as áreas e um maior conhecimento sobre o assunto. Evidências atuais em humanos corroboram estudos em modelos animais para o tratamento da obesidade através desta técnica. Porém, os estudos ainda são escassos, dificultando a formulação de recomendações para a prática profissional.

\section{The efficacy of obesity through fecal microbiota transplantation of individuals skinny}

ABSTRACT - There is a growing interest in understanding the role of the intestinal microbiota to elucidate its therapeutic potential. One of the possible application of the fecal microbiota transplantation is the treatment of obesity, also called as fecal bacteriotherapy. Thus, the objective of this study was to understand the therapeutic effect of intestinal microbiota on the health of the individual. As well as, to know the application of transplantation of fecal microbiota of lean individuals in obese individuals. It was an integrative literature review. The search for articles was carried out in April 2016, in the Regional Virtual Health Library Portal in the period 2006-2016. We used the following inclusion criteria: articles available in full free access; which addressed the study theme; presentation of summary; in Portuguese, English or Spanish. Among the descriptors of health sciences, we used the "gastrointestinal microbiome", "fecal microbiota transplantation" and "obesity". The articles showed as the main result more found that transplantation of fecal microbiota from the lean individual to obese individual can be used in the treatment of various diseases such as intestinal infections or in Diabetes Mellitus. Therefore, it is necessary to spread the subject in a multiprofessional scope facilitating increased of research in all areas and greater knowledge on the subject. Current evidence in humans confirms studies in animal models for the treatment of obesity through this technique. However, studies are still scarce, making it difficult to make recommendations for professional practice. 


\section{Introdução}

O intestino adulto abriga até 100 trilhões de bactérias residentes chamados de microbiota intestinal. Geralmente, há dois tipos de filos considerados principais em adultos, sendo ele os Bacteroidetes, compreendendo bactérias gram-negativas, e os Firmicutes, compreendendo bactérias gram-positivas (1-3).

A flora intestinal tanto de humanos como de animais pode ser modificada através da dieta. A ingestão de calorias em excesso promove a proliferação de bactérias do filo Firmicutes, que permitem a extração e estocagem de nutrientes com maior eficiência, o que auxilia no ganho de peso (4). Por outro lado, outras bactérias como Bifidobacterium, Clostridium e Bacteroides têm sido evidenciadas na melhora do metabolismo, do sistema imunitário, da resistência do câncer, da sinalização endócrina, e da função cerebral $(5,6,7)$.

Além disso, há evidências de que a comunidade microbiótica intestinal desempenha um importante papel na obesidade, especialmente as proporções de Firmicutes e Bacterioides, pois parecem ser importantes na homeostase energética do organismo, além de afetarem o sistema imune e a resposta inflamatória (8). Dessa forma, há um crescente interesse na compreensão do papel da microbiota do intestino humano para elucidar o potencial terapêutico da sua manipulação.

Alguns autores mencionam dados, ainda que limitados, de que a manipulação da comunidade microbiótica usando probióticos, antibióticos ou prebióticos podem ser utilizados no desenvolvimento de novos agentes para o tratamento da obesidade (9-10). Sendo assim, surge à alternativa que consiste no transplante de uma solução de matéria fecal a partir de um doador saudável para o trato intestinal de um receptor, com a finalidade de alterar a composição da microbiota intestinal do destinatário (11).

Tem se buscado encontrar alternativas para o tratamento da obesidade assim como o transplante da microbiota fecal (TMF), também denominado bacterioterapia fecal, no qual é transplantado comunidades bacterianas fecais de um indivíduo saudável para um indivíduo com a microbiota alterada (12). Esta hipótese é baseada em estudos que apontam que na obesidade há um desequilíbrio na microbiota do indivíduo obeso, no qual possui maior proporção do filo de Firmicutes e menor do Bacterioidetes, mostrando ainda que os mesmos se normalizam após perda de peso (13).

Em 2013, foi feita pela primeira vez a relação entre um microbioma intestinal alterado e a insulinorresistência em humanos. Com isso, através da sequenciação do metagenoma intestinal, revelou-se que as bactérias produtoras de butirato, também conhecidas pelas propriedades anti-inflamatórias, eram menos abundantes em pacientes com diabetes tipo 2 em relação a indivíduos saudáveis (14).

Deste modo, o presente trabalho torna-se necessário para que, através da literatura existente, haja um melhor entendimento a respeito de estudos que vem sendo realizados sobre o tema proposto. Contribuindo para o avanço de futuras pesquisas que promovam a saúde humana e esclarecendo as questões éticas envolvidas devido ao tema em questão envolver preceitos pessoais. $\mathrm{O}$ objetivo do presente estudo foi compreender o efeito terapêutico da microbiota intestinal na saúde do indivíduo. Assim como, conhecer a aplicação já relatada do transplante da microbiota fecal sobre doenças gastrintestinais.

\section{Material e métodos}

O presente trabalho tratou-se de uma revisão integrativa da literatura. A busca dos artigos foi realizada em abril de 2016, no Portal Regional da Biblioteca Virtual da Saúde (BVS), 
sendo delimitado o tempo da publicação dos artigos para os últimos dez anos, no período de abril de 2006 a abril de 2016. Foi escolhido este período de tempo posto que este seja um assunto pouco difundido na literatura.

Os critérios de inclusão das publicações definidos para esta revisão foram: artigos na íntegra indexados na base de dados referidos e disponíveis on-line, de acesso livre; que abordaram a temática do estudo; apresentação de resumo para primeira apreciação; procedência nacional ou internacional nos idiomas português, inglês e espanhol. Utilizaramse como descritores: "gastrointestinal microbiome", "fecal microbiota transplantation" e "obesity", todos de acordo com os descritores de ciências da saúde (DECS).

$\mathrm{Na}$ busca realizada pelo presente trabalho para revisão integrativa da literatura foram encontrados 23 artigos todos da base de dados MEDLINE. Após filtrar para todos os textos que estavam disponíveis para leitura, restaram 11 artigos nos quais foram selecionados. Destes, três foram excluídos devido aos critérios de inclusão, sendo selecionados oito artigos para esta revisão os quais foram lidos na íntegra e de forma exaustiva (Figura 1).

Para análise e interpretação dos dados, foi elaborado um instrumento com as categorias: ano da publicação, base de dados, título, objetivo e desfechos dos artigos selecionados. O processo de análise dos dados ocorreu através da interpretação do quadro a partir dos dados dos artigos, separando em categorias os artigos que se correlacionavam entre si, resultando na caracterização dos resultados.

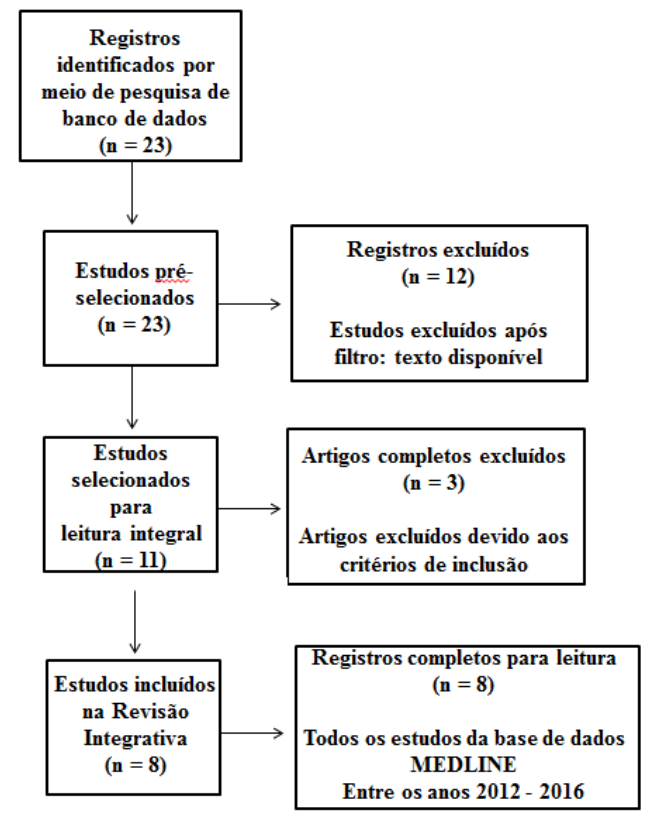

Figura 1 - Fluxograma do processo de seleção dos estudos.

\section{Resultados e discussão}

Todos os artigos encontrados foram publicados entre 2012 e 2016 e apresentam evidências moderadas e fracas sobre a temática, sendo todos de revisão e conhecimento do tema. Com isso vale salientar que o atual estudo diz respeito a um assunto recente e que necessita de mais estudos.

$\mathrm{O}$ resultado mais encontrado dentre os artigos foi que o transplante da microbiota fecal do indivíduo magro para o indivíduo obeso pode ser utilizado no tratamento de várias doenças tais como infecção por Clostridium difficile recorrente, doença inflamatória do intestino, 
síndrome do intestino irritável, prisão de ventre crônica e uma variedade de doenças não gastrointestinais como, por exemplo, Diabetes Mellitus (3,11, 15-20) (tabela 1).

A flora gastrointestinal (GI) humana é considerada um tecido, e é utilizada para transplantar a microbiota fecal no trato GI de um destinatário. A microbiota humana é estimada por conter entre 10-100 trilhões de células microbianas, e a microbiota intestinal é a maior e mais diversificada população do corpo humano (20). Em média $30 \%$ da microbiota GI são detectáveis através de técnicas, estudos de sequenciamento detalhados vem sendo realizados para conhecer as diferentes espécies bacterianas nas quais habitam o trato GI humano (21-22).

Devido à riqueza de diversidade das espécies bacterianas no intestino humano tem havido crescente interesse em entender o papel da microbiota intestinal humana e o potencial terapêutico da sua manipulação, isto pela presença particular de algumas bactérias que tem apresentado atividades bioativas (23-25).

$\mathrm{O}$ conhecimento sobre a microbiota humana tem expandido com os avanços tecnológicos de sequenciamento e as análises genômicas a respeito da diversidade na qual compõe essa microbiota intestinal e sua função. O que resultou na aplicação do TMF para uma variedade de doenças. O tratamento de TMF é a introdução de uma suspensão fecal GI derivada de um doador saudável para um receptor doente (26). O TMF é relatado, pela maioria dos autores, como de baixo custo financeiro e os resultados podem corroborar ou, até mesmo, em alguns casos substituir uma cirurgia bariátrica.

Alguns estudos encontrados na presente revisão relatam sobre a infecção por Clostridium difficile (CDI) que é uma doença GI causada por $C$. difficile, uma bactéria gram-positiva e bacilos anaeróbios produtores de toxinas (18). Os pacientes possuem a doença e que têm uma recorrência possuem até $40 \%$ de chance de uma segunda reincidência, e após a sua segunda recorrência, até $65 \%$ dos pacientes vão sofrer uma terceira (27). As altas taxas de recorrência de CDI gerou a necessidade de terapias alternativas, para o qual TMF oferece uma abordagem racional e direta. Gough et al (28) em seu estudo sobre o TMF para CDI recorrente, constatou que $89 \%$ dos 317 pacientes tiveram resolução da CDI recorrente após o tratamento com TMF.

O TMF também tem sido evidenciado como alternativa de forma eficaz para outras doenças do trato GI, como a doença inflamatória do intestino, a síndrome do intestino irritável, a prisão de ventre crônica, entre outras patologias GI. Os sintomas são caracterizados pela diarreia predominante ou constipação predominante ou, ainda, ambos os sintomas, com ausência de quaisquer anomalias anatômicas ou exames bioquímicos identificáveis (29). Estas alterações podem estar relacionadas com a modificação da microbiota intestinal e possibilidade de alívio dos sintomas e cura da doença pode ser através do TMF. Assim como demonstra em sua pesquisa Pinn et al (30) que administrou o TMF em 13 pacientes com síndrome do intestino irritável, via endoscopia digestiva alta. Encontrando $70 \%$ do alívio dos sintomas em 6 a 18 meses. Portanto, o TMF pode ter um efeito terapêutico para o tratamento de doenças GI como doenças inflamatórias.

Com relação à obesidade o presente trabalho encontrou estudos realizados com ratos obesos nos quais demonstraram uma maior capacidade de capturar calorias a partir de nutrientes no lúmen em comparação com ratos magros, isto devido ao fato da maior quantidade de bactérias do filo Firmicutes em relação ao filo Bacteroidetes (31). Além disso, o fenótipo de ratos obesos é passível de ser transmissível (32). Os autores relatam que os mesmos estudos em seres humanos são escassos, porém há indícios de que a bacterioterapia com amostra fecal melhora a sensibilidade à insulina em indivíduos diabéticos obesos (33).

Outro dado marcante na revisão integrativa da literatura está relacionado à unânime necessidade de mais pesquisas de ensaios clínicos randomizados e de coorte para estabelecer 


\section{a eficácia destes transplantes e para resultados sobre as implicações à longo prazo de manipulação das microbiotas.}

Tabela 1. Estado da arte dos artigos encontrados para revisão da literatura entre os anos 2012-2016

\begin{tabular}{ccccc}
\hline $\begin{array}{l}\text { N } \\
\text { o }\end{array}$ & Ano & $\begin{array}{c}\text { Base de } \\
\text { dados }\end{array}$ & Título & Objetivo \\
\hline & & & \\
1 & 2012 & MEDLINE & $\begin{array}{c}\text { Transplante da microbiota } \\
\text { fecal: passado, presente e } \\
\text { futuro }\end{array}$ & $\begin{array}{c}\text { Análise do aumento do conhecimento da } \\
\text { microbiota intestinal na manutenção da } \\
\text { saúde, bem como os estudos controlados de } \\
\text { TMF em uma ampla gama de distúrbios para } \\
\text { ser aceito e aplicado clinicamente. }\end{array}$ \\
& & &
\end{tabular}

22013 MEDLINE

Transplante Microbiota Fecal: indicações, métodos, evidências e os caminhos futuros

Potencial terapêutico do transplante da microbiota fecal

MEDLINE
Microbiota intestinal e diabetes tipo 2: a partir de mecanismos de percepções para perspectiva terapêutica
A microbiota intestinal e doenças não transmissíveis inflamatórios: associações e potenciais para terapias microbiota intestinal
Resumir as últimas provas, indicações e métodos de administração do TMF, e fornecer alguns insights sobre direções futuras e potenciais terapêuticos.

Revisar os atuais e melhores métodos para a realização do transplante da microbiota fecal e resumir observações clínicas que têm implicado a microbiota intestinal em várias doenças.

Demonstrar a visão geral sobre os

mecanismos subjacentes do papel da microbiota intestinal na patogênese do DM2.

Entender o papel e a influência da microbiota intestinal na saúde do indivíduo. Assim como, conhecer as terapêuticas atuais disponíveis para modular a microbiota intestinal na prevenção e tratamento de DNT; e as limitações dos métodos genéticos utilizados para estudar a microbiota intestinal humana

\section{Conclusões}

TMF restabelece a microbiota intestinal equilibrada e resulta em taxas de cura impressionantes em pacientes com CDI recorrente. Estudos recentes têm demonstrado que a patogênese de muitas doenças, tanto gastrointestinal ou não, resulta da desregulação relacionada com a microbiota.

O TMF é uma forma alternativa, sem evidência de uso de medicamentos. A sua aceitação como opção de tratamento se deve ao seu potencial terapêutico. Comprovada como a terapia mais efetiva para R-CDI. No que diz respeito ao seu papel em outras condições associadas

há escassez de dados.

O TMF parece ser um tratamento seguro e promissor para CDI recorrente. Ensaios

clínicos randomizados controlados são necessários para estabelecer a eficiência do TMF para outras doenças, e esses estudos devem analisar a composição da microbiota fecal intestinal antes e depois do TMF.

A microbiota intestinal pode desempenhar um papel importante no diabetes mellitus tipo 2, influenciando no peso corporal, no metabolismo de ácidos biliares, na atividade pró-inflamatória e resistência à insulina e modulação de hormônios do intestino. No entanto, não se sabe se as mudanças na microbiota influenciam diretamente as células $\beta$ e função das ilhotas em pacientes com DM2.

Modelos experimentais demonstram que a microbiota intestinal exerce funções nutricionais e melhora da imunidade. Os estudos observacionais mostram variações da microbiota intestinal e sua diversidade quando associada às DNT. As meta-análises usando probióticos para a prevenção de alergias demonstra redução da incidência de eczema. O TMF regula a inflamação em modelos experimentais. 
Papel da microbiota intestinal em lipídios e no metabolismo da glicose em Diabetes Mellitus
Fornecer comentário sobre a utilidade clínica dos dados da microbiota intestinal em lipídios e no metabolismo da glicose em Diabetes Mellitus
Transplante de microbiota fecal: em perspectiva

Clostridium difficile recorrente infecção e a microbiota
Apresentar estudos de interesse na compreensão do papel da microbiota intestinal humana para elucidar o potencial terapêutico do transplante fecal da microbiota
Resumir os aspectos chave do Clostridium difficile (CDI) recorrente, e introduzir o quadro e desafios do transplante da microbiota fecal, como é atualmente praticada

Não se pode inferir que as bactérias intestinais desempenham um papel causa no metabolismo da glicose e dos lipídios. Necessita de mais estudos de intervenção, incluindo o transplante fecal suplementação de estirpes bacterianas. As coortes de indivíduos saudáveis, utilizando amostras fecais coletadas no início do estudo podem ajudar a identificar específicas estirpes intestinais causalmente envolvidos bacterianas que impulsionam o metabolismo humano aberrante.

O TMF tem sido visto como um tratamento natural, de aplicação barata. Apesar disso, há dúvidas do potencial infeccioso da terapia. Pacientes tratados com TMF para

distúrbios intestinais notaram melhorara nas comorbidades à obesidade, revelando modificação da microbiota.

Mas, poucos estudos de longo prazo têm sido realizados para avaliar a segurança e o risco teórico permanece do transplante.

A TMF tem demonstrado um grande potencial no tratamento de CDI recorrente. A bacterioterapia da microbiota fecal tem liderado o caminho de alvos terapêuticos microbianos. Isto com base na evolução dos mecanismos pelos quais as comunidades microbianas asseguram proteção contra certas doenças.

TMF: Transplante da Microbiota Fecal; CDI: Clostridium difficile; DNT: Doença crônica Não Transmissível; DM: Diabetes Mellitus

\section{Conclusão}

O transplante da microbiota fecal é uma alternativa terapêutica à base de microbianos, a fim de restaurar a microbiota intestinal alterada, e têm demonstrado um grande potencial no tratamento de diferentes patologias. Evidências atuais em humanos corroboram com estudos em modelos animais para o tratamento da obesidade através desta técnica. Porém, os estudos ainda são escassos, dificultando a formulação de recomendações para a prática profissional. Portanto, se faz necessário a divulgação do tema em um âmbito multiprofissional facilitando o aumento de pesquisas em todas as áreas e um maior conhecimento sobre o assunto.

Conflito de interesses: Os autores declaram não haver conflito de interesses

\section{Referências}

1. Quin J, Li R, Raes J, Arumugam M, Burgdorf KS, Manichanh C, et al. A human gut microbial gene catalogue established by metagenomic sequencing. Nature 2010; 464: 59-65. 
2. Lozupone CA, Stombaugh JI, Gordon JI, Jansson JK, Knight R. Diversity, stability and resilience of the human gut microbiota. Nature 2012; 489: 220-230.

3. West CE, Renz R, Jenamlm MC, Kozyrskyj AL, Allen KJ, Vuillermin P, Prescott SA. The gut microbiota and inflammatory noncommunicable diseases: Associations and potentials for gut microbiota therapies. Clinical reviews in allergy and immunology 2015; 136(1): 3-13.

4. Yazigi A. Rôle de la flore intestinale dans l'insulinorésistance et l'obésité. Presse Med 2008; 10 : 1427- 1430

5. Mills K. TLR-dependent T cell activation in autoimmunity. Nat Rev Immunol 2011; 11: 807-822.

6. Tremaroli V, Backhed F. Functional interactions between the gut microbiota and host metabolism. Nature 2012; 489: 242-249.

7. Hollister E, Gao C, Versalovic J. Compositional and functional features of the gastrointestinal microbiome and their effects on human health. Gastroenterology 2014; 146: 1449-1458.

8. Qin J, Li Y, Cai Z. A metagenome-wide association study of gut microbiota in type 2 diabetes. Nature 2012; 49: 55-60.

9. Tennyson CA, Friedman G. Microecology, Obesity and Probiotics. Curr Opin Endocrinol Diabetes Obes 2008; 15: 422-427.

10. Pistelli GC, Costa CEM. Bactérias intestinais e obesidade. Revista Saúde e Pesquisa 2010; 3(1): 115-

119.

11. Gupta S, Allen-Vercoe E, Petrof EO. Fecal microbiota transplantation: in perspective. Ther Adv Gastroenterol 2016; 9(2): 229-239.

12. Khoruts A, Dicksved J, Jansson JK, Sadowsky MJ. Changes in the composition of the human fecal microbiome after bacteriotherapy for recurrent Clostridium difficile-associated diarrhea. J Clin Gastroenterol 2010; 44(5): 354-60.

13. Aggarwal J, Swami G, Kumar M. Probiotics and their effects on metabolic diseases: An update. Journal of Clinical and Diagnostic Research 2013; 7(1): 173-177.

14. Karlsson FH, Tremaroli V, Nookaew I, Bergstrom G, Behre CJ, Fagerberg B, Nieslsen J, Backhed F. Gut metagenome in European women with normal, impaired and diabetic glucose control. Nature 2013. 498(7452): 99-103.

15. Borody TJ, Paramsothy S, Agrawal G. Transplante de Microbiota fecal: Indicações, métodos, evidências e direções futuras. Curr Gatroenterol Rep 2013; 15 (337): 2-7.

16. Aroniadis OC, Brandt LJ. Transplante da microbiota fecal: passado, presente e futuro. Current Opinion in Gastroenterology 2012; 29 (1): 79-84.

17. Han JL, Lin HL. Microbiota intestinal e diabetes tipo 2: a partir de mecanismos de percepções para perspectiva terapêutica. World Journal of Gastroenterology 2014; 21 (20): 17737-17745.

18. Almeida R, Gerbaba T, Petrof EO. Recurrent Clostridium difficile infection and the microbiome. Journal Gastroenterol 2016; 51: 1-10.

19. Olden C, Groen AK, Nieuwodorp M. Papel da microbiota intestinal em lipídios e no metabolismo da glicose em Diabetes Mellitus 2015; 37 (6): 1172-1177.

20. Smits LP, Bouter KEC, Vos WM, Borody TJ, Newdorp M. Potencial terapêutico do transplante da microbiota fecal. Gastroenterology 2013; 145 (5): 946-953.

21. Eckburg PB, Bik EM, Berntein CN. Diversity of the human intestinal microbial flora. Science 2005; 308: 1635-1638.

22. Frank DN, Sant Amand AL, Feldman RA. Molecular phylogenetic characterization of microbial community imbalances in human inflammatory bowel diseases. Proc Natl Acad Sci 2007; 104: 13780-13785.

23. Gill S, Pop M, Deboy R, Eckburg P, Turnbaugh P, Samuel B. Metagenomic analysis of the human distal gut microbiome. Science 2006; 312: 1355-1359.

24. Wang Y, Hoenig J, Mallin K, Qamar S, Petrof E, Sun J. 16s rRNA gene-based analysis of fecal microbiota from preterm infants with and without necrotizing enterocolitis 2006; 3: 944-954.

25. Claesson M, Jeffery I, Conde S, Power S, O'Connor E, Cusack S. Gut microbiota composition correlates with diet and health in the elderly. Nature 2012; 488: 178-184.

26. Zhang F, Luo W, Shi Y. Should we standardize the 1,700-yearold fecal microbiota transplantation? Am J Gastroenterol 2012; 107 (11): 1755.

27. Huebner ES, Surawiez CM. Treatment of recurrent Clostrium difficile diarrrhea. Gastroenterol Hepatol 2006; 2: 203-208.

28. Gouch E, Shaikh H, Manges AR. Systematic review of intestinal microbiota transplantation (fecal bacteriotherapy) for recurrent Clostridium difficile infection. Clin Infect Dis 2011; 53 (10): 994-1002.

29. Pinn D, Aroniadis O, Brandt L. Is fecal microbiota transplantation (FMT) an effective treatment for patients with functional gastrointestinal disorders (FGID)? Neurogastroenterol Motil 2015. 27: 19-29. 
30. Pinn D, Aroniadis O, Brandt L. Follow-up study of fecal microbiota transplantation (FMT) for the treatment of refractory irritable bowel syndrome (IBS). Presented at the American College of Gastroenterology Annual Meeting; San Diego, CA, abstract 2013: P1688.

31. Sekivovi I, Russell SL, Antunes CM. Gut microbiomes in health and disease. Physiol Rev 2010; 90: 859-904.

32. Turnbaugh PJ, Ley RE, Mahowakd MA. An obesity-associated gut microbiome with increased capacity for energy harvest. Nature 2006; 444: 1027- 1031.

33. Vrieze A, Nood EV, Holleman F, Salojarvi J, Kootte RS, Bartelsman JFWM. Transfer of intestinal microbiota from lean donors increases insulin sensitivity. Gastroenterology 2013; 144: 19-20. 